\title{
Observations of Microwave Continuum Emission from Air Shower Plasmas
}

\author{
P. W. Gorham, ${ }^{1}$ N. G. Lehtinen, ${ }^{1,}$ * G. S. Varner, ${ }^{1}$ J. J. Beatty, ${ }^{2}$ A. Connolly, ${ }^{3}$ \\ P. Chen, ${ }^{4}$ M. E. Conde, ${ }^{5}$ W. Gai,${ }^{5}$ C. Hast,${ }^{4}$ C. L. Hebert,,${ }^{1}$ 月 C. Miki, ${ }^{1}$ R. Konecny, ${ }^{5}$ \\ J. Kowalski, ${ }^{1}$ J. Ng, ${ }^{4}$ J. G. Power, ${ }^{5}$ K. Reil, ${ }^{4}$ D. Saltzberg, ${ }^{3}$ B. T. Stokes, ${ }^{1,}$ 用 and D. Walz ${ }^{4}$ \\ ${ }^{1}$ University of Hawaii at Manoa, Department of Physics and Astronomy, Honolulu, Hawaii 96822 \\ ${ }^{2}$ Ohio State University, Dept. of Physics, Columbus, OH. \\ ${ }^{3}$ Univ. of Calif. at Los Angeles, Dept. of Physics, Los Angeles, CA \\ ${ }^{4}$ Stanford Linear Accelerator Center, 2575 Sand Island Rd., Menlo Park, CA. \\ ${ }^{5}$ Argonne National Laboratory, Argonne, IL.
}

\begin{abstract}
We investigate a possible new technique for microwave detection of cosmic ray extensive air showers which relies on detection of expected continuum radiation in the microwave range, caused by free-electron collisions with neutrals in the tenuous plasma left after the passage of the shower. We performed an initial experiment at the AWA (Argonne Wakefield Accelerator) laboratory in 2003 and measured broadband microwave emission from air ionized via high energy electrons and photons. A follow-up experiment at SLAC (Stanford Linear Accelerator Center) in summer of 2004 confirmed the major features of the previous AWA observations with better precision. Prompted by these results we built a prototype detector using satellite television technology, and have made measurements suggestive of detection of cosmic ray extensive air showers. The method, if confirmed by experiments now in progress, could provide a high-duty cycle complement to current nitrogen fluorescence observations.
\end{abstract}

\section{INTRODUCTION}

The origin and nature of the ultra-high energy cosmic rays (UHECR) remains one of the enduring mysteries of experimental particle astrophysics. In spite of well over four decades of observations of $10^{20} \mathrm{eV}$ UHECR by many different experiments [1], we still do not have a confirmed astrophysical source for these particles, nor do we understand their composition in any detail, nor do we know how they propagate from their unknown sources to earth [2]. In the last decade new observatories such as HiRes and most recently the Auger Observatory have much improved the statistics on measurements of these particles, but the issues of their origin and propagation remain largely open. As the highest energy subatomic particles observed in nature, UHECRs must arise from the most energetic phenomena in our universe. Their study is thus crucial to understanding the nature of acceleration processes that can attain energies some seven orders of magnitude higher than is currently achievable in the laboratory [3, 4, 5].

The primary UHECR spectrum is described by a simple power law $J(E) \propto E^{-\alpha}$ with $\alpha \simeq 2.7$ for $10^{18.5}<E<$ $10^{19.5} \mathrm{eV}[6,18]$. Above $10^{19.5}$, the interaction length of cosmic ray nucleons on the cosmic microwave background becomes comparable to intergalactic separation distances, a process first described by Greisen [8], and Zatsepin \& Kuzmin [9] and now known as the GZK process. It is precisely at and above the GZK energies that the measurements of the primary UHECR become uncertain due to low statistics, and the shape of the spectrum near the endpoint is still a subject of active debate.

Because of the scarcity of particles at these highest en-

\footnotetext{
* currently at Stanford University

${ }^{\dagger}$ currently with Raytheon Corp., Tucson, AZ

${ }^{\ddagger}$ currently at John A. Burns School of Medicine, UH Manoa
}

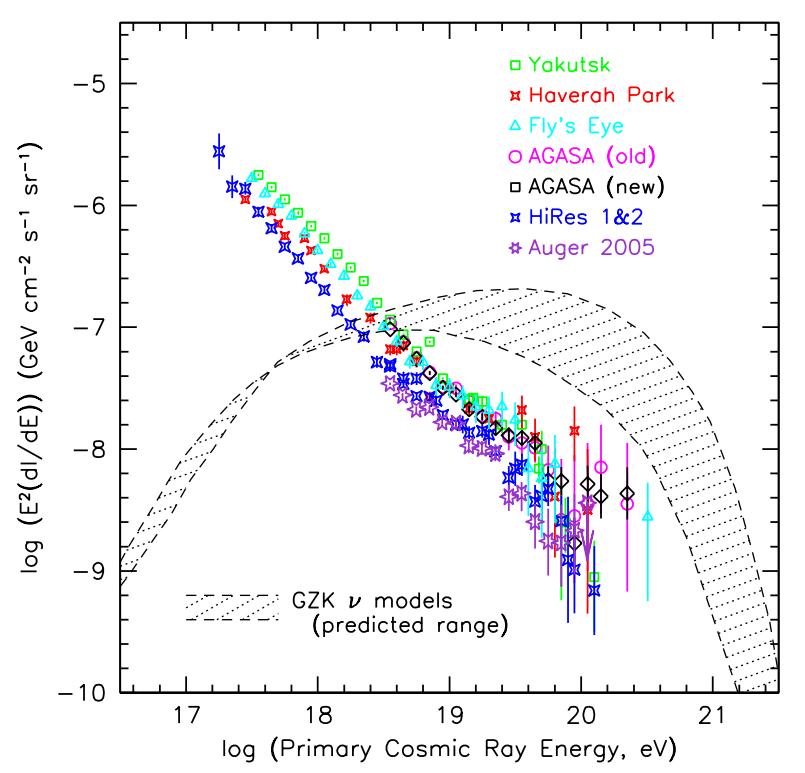

FIG. 1: World ultra-high energy cosmic ray and predicted cosmogenic neutrino spectrum as of early 2007, including data from the Yakutsk [13], Haverah Park [14] the Fly's Eye [16], AGASA [7], HiRes [18], and Auger [26], collaborations. Data points represent differential flux $d I(E) / d E$, multiplied by $E^{2}$. Error bars are statistical only. GZK neutrino models are from Protheroe \& Johnson [20] and Kalashev et al. [21].

ergies, research into new methods has focused on indirect means of observation [24, 25], which makes use of radiated air-fluorescence emission from the air shower to observe it at distances of up to tens of $\mathrm{km}$ from the particle axis. By observing the longitudinal and transverse development of UHECR-induced extensive air showers (EASs) investigators are gaining information on the primary composition, which favors light elements and disfavors a significant electromagnetic 
(e.g. photon) component. Such studies can also elucidate the high energy physics of the early interactions, which occur at center-of-mass energies well above that currently probed by accelerators[2].

The region near the endpoint of the UHECR energy spectrum is shown in summary form in Fig. 1, where no effort has been made to correct the systematic offsets in the flux levels of the different experiments involved. Above $10^{20} \mathrm{eV}$, the event rate is of order 1 per $\mathrm{km}^{2}$ per century, producing still only a handful of events per year close to this threshold in all existing UHECR observatories. As is apparent from the current world spectrum, constraints on the high energy tail or statistically compelling details of any putative cutoff above the current highest energies will still require years of observation. The need for much-improved statistics to address the primary issues currently under investigation all argue for expansions of and improvements on existing methods. Such issues include the detailed shape of the UHECR energy spectrum [6, 7] (including the presence, or lack thereof, of the GZK cutoff [8, 9]), energy-resolved primary particle composition [10, 11], and source production mechanisms (i.e. origins) [12, 17, 22, 23].

In addition, the virtual certainty of the extragalactic origin of these particles ensures an associated cosmogenic neutrino flux, generated via photohadronic processes throughout the universe [19]. Hadronic cosmic rays above $\sim 10^{19} \mathrm{eV}$ propagating in the $2.7 \mathrm{~K}$ cosmic microwave background radiation (CMBR) exceed the threshold for resonant $\Delta^{+}$particle production through the GZK process, and the rapid decay of these unstable secondaries leads to pions and subsequently neutrinos. The mean free path of a $10^{20} \mathrm{eV}$ proton in the CMBR is several Mpc in the current epoch, whereas the neutrinos are unattenuated from any cosmic distance. Future observations of cosmogenic GZK neutrinos will provide a unique and complementary view of the UHECR production, propagation, and attenuation throughout the universe, motivated by the UHECR observations themselves. Figure 1 shows also a band indicating the range of models for these cosmogenic neutrinos. The uncertainties in the models stem primarily from the details of the highest energy part of the UHECR spectrum, as well as the epoch of maximum UHECR source evolution, and GZK neutrino observations will thus provide independent constraints on the UHECR sources.

\section{Motivation for Microwave EAS Detection.}

While there is general agreement among the different experiments as to the global properties of the UHECR spectrum, as Figure 1 shows, there is still significant disagreement and uncertainty on absolute flux scales and on some of the fundamental questions of UHECR research. The two primary techniques of UHECR observation, ground-based particle arrays and optical fluorescence detectors both suffer from tangible limitations. In the case of ground arrays, only a single slice of EAS longitudinal development can be observed. This means that determinations of primary particle energy and composition require extrapolation via model-dependent estimates, which may disagree depending on the model used. While the optical fluorescence method enables one to observe longitudinal as well as transverse shower development, it is highly constrained by the fact that it can only work on clear, moonless nights. This leads to a net yearly duty cycle of only 5-10\%[24]. Furthermore, because the highest energy events are observed at increasingly large distances, even small fluctuations in atmospheric aerosol contamination can have substantial effects on energy estimation.

An air shower dissipates virtually its entire energy budget through ionization, producing a tenuous plasma with an electron temperature of order $10^{5} \mathrm{~K}$ or more. The ionization and subsequent de-excitation of molecular nitrogen in the $\mathrm{N}_{2}^{+*} 1 \mathrm{~N}$ and $2 \mathrm{P}$ states leads directly to the optical $\mathrm{N}_{2}$ fluorescence now observed. The hot air shower plasma cools rapidly on 1-10 nanosecond time scales, distributing its thermal energy through collisions with the neutral molecules, primarily $\mathrm{N}_{2}$, which has the largest cross section and number density. This rapid cooling process leads to additional excitation of rotational, vibrational, electronic valence, and other modes of kinetic energy distribution among molecules, many of which can also lead to rapid de-excitation and subsequent emission.

In turn, the hot electrons themselves, while producing this excitation, can produce their own emission, such as continuum bremsstrahlung emission, or recombination radiation. The fraction of total radiated energy in optical fluorescence, compared to the total available energy budget for secondary radiation, is very small, leaving much possible radiative energy still unaccounted for. The possibilities for observing secondary air shower plasma emission other than optical fluorescence have not yet been explored in any detail.

We report here on investigations of the feasibility of other channels for EAS observations. To this end we have undertaken several experimental efforts, including two accelerator experiments. The promising results from these measurements have led us to commission a testbed prototype detector, which has helped to establish the methodology that could be used to make detailed measurements of EAS microwave molecular bremsstrahlung radiation (MBR)[28]. In this report we describe the accelerator results and the testbed development that has resulted from them, which we have dubbed the Airshower Microwave Bremsstrahlung Experimental Radiometer (AMBER).

\section{A. Molecular Bremsstrahlung Radiation.}

MBR in weakly ionized air is created by free electrons accelerating through collisions with the fields of molecules in the ambient medium. EAS ionization is considered "weak" since the interactions of free electrons or ions are dominated by collisions with neutrals rather than other ions. MBR has been classically treated as a thermal process, with the emission coming from $\leq 10 \mathrm{eV}$ electrons assumed to be distributed with isotropic Maxwellian velocities. By these assumptions steady-state MBR emission is expected to be isotropic and unpolarized, which strongly differentiates it with the highly directional bremsstrahlung from relativistic particles which may 

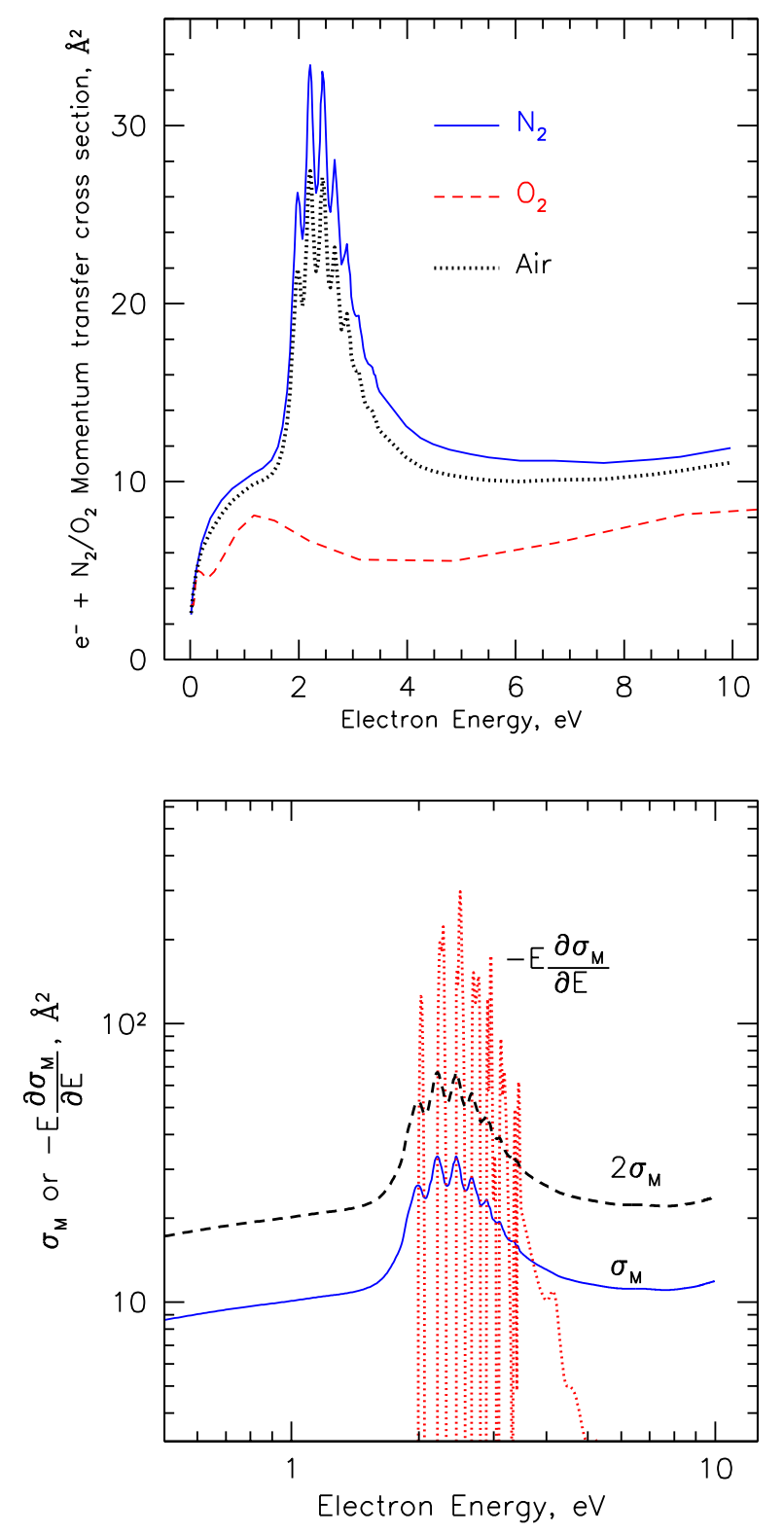

FIG. 2: Top: Cross section for momentum transfer between electrons and $\mathrm{N}_{2}, \mathrm{O}_{2}$, and air over the energy range of interest for molecular bremsstrahlung production [38, 39]. Bottom: The red-dotted lines show the function $-E \frac{\partial \sigma_{M}}{\partial E}$ which must be greater than either $\sigma_{M}$ (weak criterion; blue line) or $2 \sigma_{M}$ (strong criterion, black-dash line) as a necessary condition for stimulated bremsstrahlung emission in an air plasma. See text for details.

be more familiar to a high energy or cosmic-ray physicist. MBR emission shares this property with optical fluorescence emission, an important feature which allows for the possibility of performing shower calorimetry by mapping the MBR intensity (and thus the ionization content) along a shower, much as optical fluorescence detections maps the nitrogen excitation along the same shower. As indicated in Fig. 10, MBR emission and optical fluorescence both are emitted in all di- rections around an EAS, and detectors may therefore "image" the air shower glow as a track along the sky to establish twodimensional angular geometry, and use the timing information for the pulse arrival to determine the range evolution of the shower, giving a complete reconstruction of both geometry and particle number evolution.

The expected isotropic behavior of MBR is also in sharp contrast to relativistic radio emission processes such as Cherenkov, transition, or synchrotron radiation, all of which are beamed and highly polarized. In this respect it is convenient to think of MBR emission as analogous to "radio fluorescence," whereas beamed relativistic radio emission is closely aligned to the particle content of a shower and thus should be identified as concordant with the information derived from a ground EAS detector array. Furthermore, MBR intensity is expected to be proportional to the EAS ionization rate, which is known to be itself proportional to $N$, the total number of charged particles in the shower. This therefore leads to a direct relationship between MBR intensity and shower energy, with the degree of proportionality determined by the details of local correlations between electron velocities or radiative transitions in the tenuous air shower plasma.

The proportionality will depend on important details which require empirical determination, much the same way that oscillator line strengths necessary to understanding optical fluorescence must be determined via laboratory calibration with additional corrections for atmospheric conditions. For example, since an EAS produces an initial distribution of ionization which is likely to be a power-law rather than a Maxwellian, there are corrections for non-thermal effects such as stimulated emission and other non-equilibrium continuum radio emission channels, which may significantly increase the emission power over the minimal thermal MBR baseline. In addition, the cross sections for both elastic and inelastic collisions of electrons with air molecules are complex functions of electron energy, yielding strong velocity dependence in the electron collision frequency which can also contribute to MBR emission coefficients. Such effects are difficult to analytically estimate and will be best calibrated in situ as has been done for other EAS observation methods.

To analytically determine the expected minimum flux density for MBR, we start with the emissivity from classical bremsstrahlung analysis of collisions between electrons and neutral molecules [28]:

$$
\eta_{\omega}(\mathrm{u})=\frac{e^{2}}{16 \pi^{3} \varepsilon_{0} c^{3}} \mathrm{u}^{2} \mathrm{v}_{e n}(\mathrm{u}) \zeta\left(\mathrm{v}_{e n}, \omega\right) \text {. }
$$

Where $\omega$ is the microwave radian frequency, $u$ is the electron velocity, $\mathrm{v}_{e n}(\mathrm{u})$ is the velocity-dependent electron-neutral collision frequency, and $\zeta\left(v_{e n}, \omega\right)$ is a term that accounts for the collisional suppression of radiation which arises from the destructive interference of fields from successive collisions within the radiation formation zone of each collision, a process also known as plasma dispersion [28].

Under the assumption of an isotropic and time-stationary velocity distribution,

$$
\zeta\left(\mathrm{v}_{e n}, \omega\right)=\frac{1}{1+\left(\mathrm{v}_{e n}(\mathrm{u}) / \omega\right)^{2}} .
$$


For an altitude of $5 \mathrm{~km}, v_{e n} \simeq 3 \mathrm{THz}$ at electron energies of about $2 \mathrm{eV}$, near the peak of the collision cross section; for room temperature electrons, $v_{e n} \simeq 40 \mathrm{GHz}$. The corresponding suppression factors are $\zeta \simeq 5 \times 10^{-5}$, and $\zeta \simeq 0.4$, respectively, showing the wide extremes of values possible under the range of electron temperatures that obtain in an air shower.

To preface further discussion below, we note that there are several other effects that compete with plasma dispersion and will tend to enhance the emissivity, or "suppress the suppression." First, stimulated emission, even at a very small level, leads to correlations in electron-photon transitions. Such emission does not require a full-blown population inversion, as in laser processes. Rather, deviations from the ground-state Maxwellian distribution can enable low levels of stimulated emission. Second, velocity correlations of the electrons due to the imposed geometry of the shower tracks and the anisotropic distribution of ions can impose some cylindrical symmetry in the distributions, in tension with the assumption of uncorrelated isotropy in the electron behavior. Finally, weak plasma correlations on the scale of the Debye length can lead to coherent motion of electrons over very small scales, but large enough to overcome some fraction of the suppression effects.

To introduce an ad hoc accounting for the sum of all such effects, we impose a coherence factor $\xi$ which modifies the $\zeta$ term:

$$
\zeta_{c}\left(v_{e n}, \omega, \xi\right)=\frac{\xi}{1+\left(v_{e n}(\mathrm{u}) / \omega\right)^{2}} .
$$

where $\xi>1$ parameterizes the level of excess emission above the "suppression floor" determined by $\zeta$ in the absence of any correlation of either electrons or electron-photon transitions. The term $\xi$ can then be determined empirically to set the scaling of the emission, with $\xi=1$ representing the MBR floor level.

The emissivity $\eta_{\omega}$ above must be integrated over the distribution of electron velocities to yield the emission coefficient $j_{\omega}\left(\mathrm{W} \mathrm{m}^{-3}(\operatorname{radian} / \mathrm{s})^{-1}\right.$ steradian $\left.^{-1}\right)$ :

$$
j_{\omega}=\int_{0}^{\infty} \eta_{\omega}(\mathrm{u}) f(\mathrm{u}) \mathrm{u}^{2} d \mathrm{u},
$$

where $f(\mathrm{u})$ is the electron distribution function, which is Maxwellian in the thermal limit:

$$
f(\mathrm{u})=\left(\frac{m_{e}}{2 \pi k T_{e}}\right)^{\frac{3}{2}} \exp \left(\frac{-m_{e} \mathrm{u}^{2}}{2 k T_{e}}\right) .
$$

for electron temperature $T_{e}$. Similar analysis yields the absorption coefficient $\alpha_{\omega}$ :

$$
\alpha_{\omega}=-\frac{4 \pi}{3 c} \frac{\omega_{p}^{2}}{\omega^{2}} \int_{0}^{\infty} v_{e n}(\mathrm{u}) \zeta(\mathrm{v}, \omega) \frac{\partial f(\mathrm{u})}{\partial \mathrm{u}} \mathrm{u}^{3} d \mathrm{u}
$$

where the plasma frequency is given by $\omega_{p}^{2}=N_{e} e^{2} /\left(m_{e} \varepsilon_{0}\right)$ for electron number density $N_{e}$. These coefficients are combined to form the source function $S_{\omega}=\left(1 / n^{2}\right) j_{\omega} / \alpha_{\omega}$ which is then integrated along a ray $s$ through plasma column to the observer to determine the net intensity per unit radian frequency, or flux density $I_{\omega}$ :

$$
I_{\omega}=\int_{0}^{\tau_{0}} S_{\omega}(\tau) e^{-\tau} d \tau
$$

where the optical depth $\tau$ is defined by $d \tau=-\alpha_{\omega} d s$. Note that the absorption coefficient is not necessarily positive definite: under conditions where $\frac{\partial f(\mathbf{u})}{\partial u}>0$, e.g., if there is an inflection in the electron velocity distribution function, then $\alpha_{\omega}$ can become negative and stimulated emission will cause the radiation to grow with propagation distance.

The MBR flux density received by a ground detector is estimated by integrating the intensity thus derived over the solid angle of the receiving antenna beam to yield Watts per $\mathrm{m}^{2}$ per $\mathrm{Hz}$ over a given frequency band. The minimum detectable change in flux density for a radio antenna and receiver is [33]

$$
\Delta I_{\omega, \min }=\frac{k_{B} T_{s y s}}{A_{e f f} \sqrt{\Delta t \Delta v}}
$$

where $T_{s y s}$ is the noise temperature of the receiver system, $A_{\text {eff }}$ is the effective area of the antenna, $\Delta t$ is the receiver sampling time constant, and $\Delta v$ is the receiver bandwidth. After passage of the relativistic shower front, the emission continues during the electron thermalization time, $t_{t h}$. This time is determined by both elastic and inelastic collisions of electrons with air molecules [36, 37], giving $t_{t h} \simeq 10 \mathrm{~ns}$ for dry air at 1 atmosphere. The received radiation continues during the time the shower remains in the field-of-view of the antenna. At 4 $\mathrm{GHz}$, for a $D=1.8 \mathrm{~m}$ dish (giving a beamwidth $2 \lambda / D \simeq 5^{\circ}$ ), this time can be many microseconds for a distant shower.

Note that there is also a direct analog possible between an optical fluorescence detector which used photomultiplier "pixels" to image the two-dimensional projection of the optical fluorescence intensity along the shower, and a radio dish which can use an array of focal plane receiver feeds (each of which is effectively a pixel) to image the MBR intensity along the same shower. Feed pixel-arrays are not so common in radio astronomy because of the success of radio interferometry, but they are established technology where low-resolution bolometric imaging is important (for example, in submillimeter radio astronomy).

Based on the parameters assumed above, we have numerically integrated the flux density for air showers at a distance of $10 \mathrm{~km}$, and we find that the MBR floor level of emission, including the full suppression term given in equation ?? above with $\xi=1$, gives an average detection energy threshold of order $10^{19} \mathrm{eV}$. However, as we have already noted, estimates of the MBR emission of UHECR air showers using the standard thermal electron formalism here indicate that the energy threshold for detectability of the emission may depend strongly on the behavior of the modified suppression term $\zeta_{c}$, which is affected by several classes of non-equilibrium conditions that can obtain in an air shower plasma. We address these in the next section. 


\section{Departures from Equilibrium conditions.}

A summary of the conditions under which departures from the MBR floor are expected is given in Table I. Each of these conditions may play a role in air shower emission, and we discuss each of them in turn.

TABLE I: Assumptions for the standard MBR derivation compared to actual conditions in air showers.

\begin{tabular}{|l|l|}
\hline $\begin{array}{l}\text { Assumed Condition, standard } \\
\text { MBR derivation }\end{array}$ & $\begin{array}{l}\text { Actual conditions for air } \\
\text { shower plasma }\end{array}$ \\
\hline $\begin{array}{l}\text { Maxwellian (thermal) elec-- } \\
\text { tron speeds }\end{array}$ & $\begin{array}{l}\text { Non-thermal, cascade power- } \\
\text { law with high-energy tail }\end{array}$ \\
\hline $\begin{array}{l}\text { Isotropic velocity and mo- } \\
\text { mentum distribution }\end{array}$ & $\begin{array}{l}\text { Linear ion-trails introduce } \\
\text { first-order anisotropy }\end{array}$ \\
\hline $\begin{array}{l}\text { Time-stationary, in thermal } \\
\text { equilibrium }\end{array}$ & $\begin{array}{l}\text { Highly non-stationary, fast- } \\
\text { transient relaxation }\end{array}$ \\
\hline $\begin{array}{l}\text { Collision frequency a weak } \\
\text { function of electron speed }\end{array}$ & $\begin{array}{l}\mathrm{N}_{2} \text { cross section a strong } \\
\text { function of electron speed }\end{array}$ \\
\hline
\end{tabular}

a. Stimulated Bremsstrahlung. As an example of the departure from the assumptions regarding the velocity dependence of the electron collision frequency, Figure 2(top) shows the experimental electron-molecular nitrogen momentum transfer cross section $\sigma_{M}$ in the energy range of interest. Most notable is the $2.3 \mathrm{eV}$ resonance due to elastic collisions that lead to rotational excitation [38]. This resonance region is in fact the complement of what is observed in optical fluorescence-the energy transferred in this region of the cross section is released in part through optical fluorescence, and the complexity of it is in part mirrored in the structure of the optical nitrogen fluorescence transitions. Such highly nonlinear changes in collisional parameters with electron energy strongly depart from the assumptions used above, and similar effects have been found to lead to stimulated emission even in highly collisional plasmas under some conditions [28].

In fact, stimulated bremsstrahlung emission from gas discharge plasmas was observed in the 1980's in a series of experiments [29, 31? ]. Necessary, though not sufficient, criteria can be stated for stimulated bremsstrahlung emission:

$$
-E \frac{\partial \sigma_{M}}{\partial E}>\sigma_{M}
$$

for anisotropic electron distributions whose direction is parallel to the direction of the electric vector of the propagating radiation, and

$$
-E \frac{\partial \sigma_{M}}{\partial E}>2 \sigma_{M}
$$

for isotropic electron distributions. Figure 2 (bottom) evaluates this condition for the molecular nitrogen case shown in Fig. 2(top), and it is evident that both of the conditions above are strongly satisfied in the neighborhood of the resonance. Under such non-equilibrium conditions, an electron population inversion in the ionized region is possible, and this can lead potentially to stimulated emission [28]. Such inverted populations have been observed in discharge experiments in molecular nitrogen plasmas [34, 35].

b. Oxygen attachment. Molecular oxygen has a momentum transfer cross section significantly lower than that of nitrogen over this energy range, as shown in Fig. 2(top), and, after weighting for abundance, its effect on the overall momentum transfer cross section in air is minimal. Although $\mathrm{O}_{2}$ does not contribute much to the thermalization of electrons in air, it does however play an extremely important role in removing free electrons once they have thermalized, since the three-body attachment cross section to $\mathrm{O}_{2}$ rises steeply at low electron energies. In fact the attachment time scale for room temperature electrons is comparable to the $\sim 10 \mathrm{~ns}$ thermalization time scale for hot electrons in 1-atmosphere air [41]. Once attached to ions, the electrons can no longer contribute to the bremsstrahlung continuum radiation. And since the initial spatial distribution of the oxygen ions is highly structured, and the ions are almost stationary in the short period during electron attachment, they impose a rapidly developing anisotropy in the removal of electrons from the free distribution, creating MBR in the free-bound transition. This effect also imposes anisotropy in the velocity distribution of the electrons.

c. Plasma correlations. The Debye length $\lambda_{D}$, over which an electron is fully shielded from a neighboring ion in a plasma, is given by

$$
\lambda_{D}=\sqrt{\varepsilon_{0} k T_{e} /\left(e^{2} n_{e}\right)}
$$

where $n_{e}$ is the electron number density. For an EAS plasma at an energy of $10 \mathrm{EeV}$ or more, $\lambda_{D} \sim 1-2 \mathrm{~cm}$. Since over this distance there may be several hundred ion pairs along each relativistic through-going particle track, along with several hundred to several thousand tracks per square $\mathrm{cm}$ in the vicinity of the EAS core, electrons do not behave entirely independently but are subject to weak bulk plasma effects at some level which will produce phase-space correlations. For our case we may class these effects together with the attachment effects above; in either case the end result will be parameterized via equation 3 .

\section{Radiative Coherence.}

While the field strength for a single electron is accurately described by the MBR theory, the summation of these fields in the presence of correlated velocities can significantly alter the resulting ensemble field strength. Such alterations, which may be produced by intrinsic shower geometry, or electron-photon correlations from stimulated emission, or by other plasma effects, still may be described via simple vector sums of the field strength of each of the radiating particles involved.

For individual emitters the resultant field strength will grow as a phasor sum [42]:

$$
\vec{E}=\sum_{j=1}^{N_{e}} \overrightarrow{\varepsilon_{1}}(\mathbf{v}) \exp \left(-i \vec{k} \cdot \vec{x}_{j}\right)
$$


where $N_{e}$ is the total number of electrons in the plasma, $\overrightarrow{\varepsilon_{1}}(\mathbf{v})$ is the field radiated from a single electron, $\vec{k}$ is the wave vector of the radiation, and $\vec{x}_{i}$ is is the position of the $j$ th electron with respect to the observation point. The total radiated farfield power per unit area $P / A$ is given by the magnitude of the Poynting flux

$$
P / A=\left|S_{t o t}\right|=|\vec{E}|^{2} / Z_{0}
$$

where $Z_{0} \simeq 377 \Omega$ is the impedance of free space. In the limit of complete coherence, the phase factors $\vec{k} \cdot \vec{x}_{i}$ are all unity, $|\vec{E}|=N_{e} \varepsilon_{1}$, and the total coherent power is $P_{c o h}=N_{e}^{2} P_{1}$, where $P_{1}$ is the power radiated from a single electron. Since $N_{e}$ is proportional to shower energy, the coherent power depends quadratically on the energy of the primary particle. In the incoherent limit, the sum of the phase factors corresponds to a two-dimensional random walk in the real and imaginary components of the resultant field strength, and the total power grows as $P_{\text {incoh }}=N_{e} P_{1}$.

While in general the partially coherent case requires a detailed knowledge of the electron phase space distribution function, we can get a qualitative sense of the behavior by considering a case where the $N_{e}$ electrons consist of $M$ subgroups of $\mu_{e}$ electrons each, such that $N_{e}=M \mu_{e}$. Assume that the $\mu_{e}$ electrons in each subgroup radiate coherently, but that the subgroups themselves are uncorrelated. Thus, while the radiated fields from the $M$ subgroups add incoherently, the subgroup electrons themselves radiate coherently, and the resulting partially coherent power is $P_{\text {part }}=M \mu_{e}^{2} P_{1}$, now quadratic in $\mu_{e}$ rather than $N_{e}$. The ratio of the partially coherent power to the incoherent power is proportionally

$$
\frac{P_{\text {part }}}{P_{\text {incoh }}}=\frac{M \mu_{e}^{2} p_{i}}{N_{e} p_{i}}=\mu_{e}
$$

Similarly the ratio of coherent-to-incoherent power grows as $N_{e}$. Since the plasma density of ionization electrons in a shower scales linearly with shower energy, both the coherent and partially coherent regimes will yield radiated power that grows quadratically with shower energy. In fact, as soon as $\mu_{e} \geq 10$, coherence begins to dominate over the incoherent component by an order of magnitude or more. Even modest correlations among the shower ionization electrons can thus rapidly lead to much larger detected emission than expected.

We have parameterized these effects using the correction term $\xi$ which modifies the collisional decoherence factor $\zeta$ as described above. In practice empirical data will be required to establish the emission constants associated with these factors, as is the case for all other emission mechanisms in a real air shower.

\section{B. Accelerator beam tests.}

Motivated by the fact that even the floor level of fullysuppressed emission from the MBR process appeared to us to be detectable under air shower plasma conditions, we have performed two accelerator tests designed to measure the MBR in a laboratory air-shower plasma. In these experiments we have found good evidence for microwave continuum emission with characteristics suggestive of a major departure from the standard incoherent MBR emission scenario, not an unexpected result given the variety of different non-equilibrium, non-thermal, and partially coherent processes that are possible. We detail these results here.
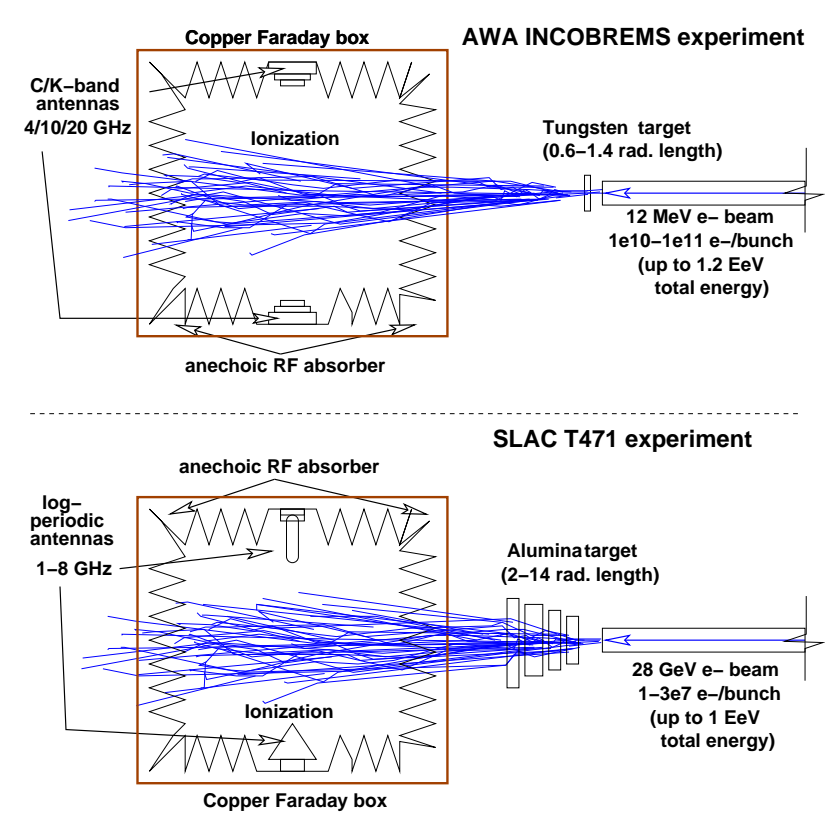

FIG. 3: Schematic of AWA INCOBREMS (top) and SLAC T471 (bottom) experiments, which used electron beams to shower in either Tungsten or alumina targets to produce ionization inside an anechoic Faraday chamber, observed by internal antennas.

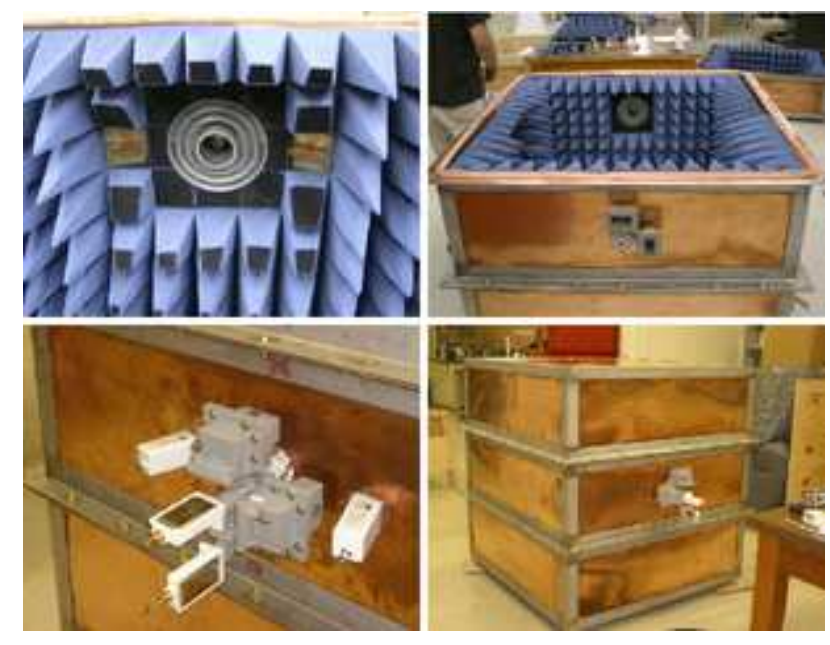

FIG. 4: Views of the exterior and interior of the Faraday anechoic chamber used for measurements of microwave continuum emission in the INCOBREMS and T471 experiments. The box is approximately a $1 \mathrm{~m}$ cube in dimensions. 

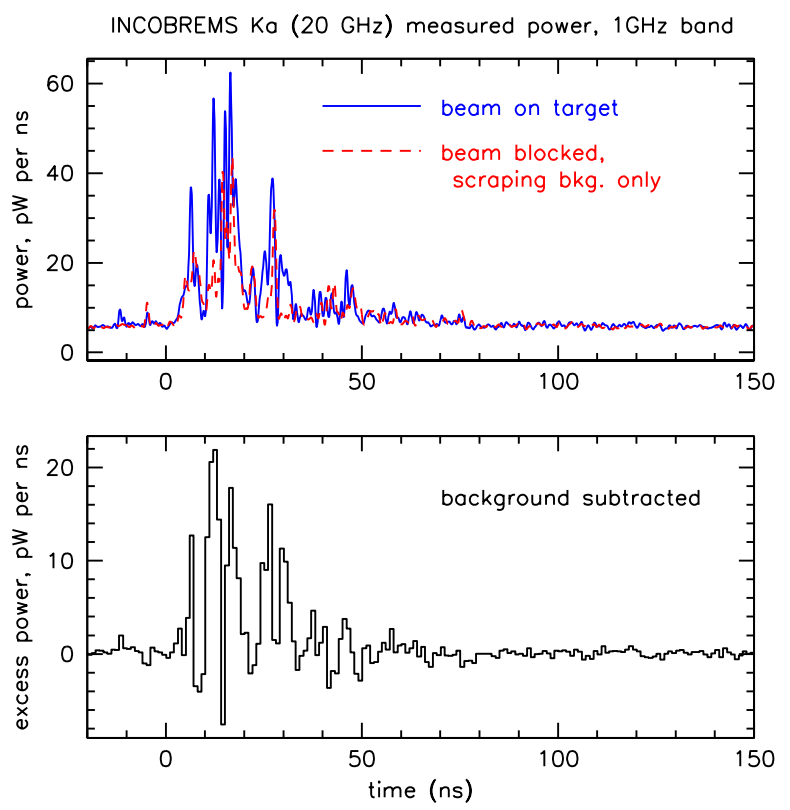

FIG. 5: Phase-stable (partially coherent) component for $20 \mathrm{GHz}$ emission observed from the $5 \mathrm{~mm}$ tungsten target, where background subtraction of beam-scraping backgrounds was possible. Upper pane shows blue as total emission and red as the background due to stray ionization from the beam; the lower pane show the backgroundsubtracted results. The data is averaged over several thousand beam shots.

\section{AWA INCOBREMS.}

In June 2003, the INCOBREMS experiment was performed at the Argonne Wakefield Accelerator (AWA). The beam used for the experiment consisted of $12 \mathrm{MeV}$ electrons, pulsed in $\sim 7 \mathrm{nC}$ charge bunches of $1.2 \mathrm{~cm}$ longitudinal thickness (40 ps duration) containing $\sim 4 \times 10^{10}$ electrons. This gives a typical total beam energy of $5 \times 10^{17} \mathrm{eV}$. The beam was collided into a fixed radiator of tungsten with a thickness of 2 or $5 \mathrm{~mm}$ (tungsten has a radiation length of $3.5 \mathrm{~mm}$ ), producing a core of photons with energies $5-10 \mathrm{MeV}$ as well as some lower energy electrons. Typically $40-90 \%$ of the total energy was extracted into photons that traversed the chamber, depending on the radiator. The conversion was necessary since the $12 \mathrm{MeV}$ electron energy was well below the critical energy in air, and thus inadequate to produce a fully developed shower. By converting to gamma-rays we avoided a large excess negative charge associated with the passage of the electron beam through the Faraday chamber. The photons entered an air-filled $\sim 1 \mathrm{~m}^{3}$ copper anechoic Faraday chamber which prevented interference from outside electromagnetic radiation and absorbed transition radiation caused by beam effects on the copper. C (3.4-4.2 GHz), Ku (10.7-11.8 GHz) and $\mathrm{Ka}(20.2-21.2 \mathrm{GHz})$ band commercial radio receivers were mounted on the insides of the chamber to measure subsequent radio emissions. Figure 3 shows a schematic view of the general layout for both this and the subsequent SLAC experiments, and Fig. 4 shows several views of the anechoic
Faraday chamber employed in both cases (here shown with the antennas and receivers used for the INCOBREMS experiment).

The photon bunches in the AWA experiment typically deposited about $1 \mathrm{PeV}$ of ionization energy while passing through the Faraday chamber. Since the radiation length of electromagnetic particles in air is of order $300 \mathrm{~m}$ at sea level, the deposited energy is of order

$$
E_{\text {chamber }} \sim \frac{1}{300}\left(1-\frac{1}{e}\right)\left(5 \times 10^{17} \mathrm{eV}\right) \simeq 10^{15} \mathrm{eV} .
$$

The mean energy required per ion pair is of order $30 \mathrm{eV}$, and there are thus about $3 \times 10^{13}$ ion pairs produced in the chamber for each beam shot. The distribution of the free electrons in the plasma is of course much denser than the equivalent $\mathrm{PeV}$ cosmic ray air shower. Most $(\sim 80 \%)$ of the plasma is produced in a central cylindrical region through the chamber, with a radius of order $25 \mathrm{~cm}$, and a mean plasma density of order $10^{8} \mathrm{e}^{-} \mathrm{cm}^{-3}$.

Based on the expectations of MBR, we expected to observe emission that was incoherent, with intensity that scaled linearly with beam current. When our initial observations indicated that the emission appeared to be scaling coherently, with intensity going as the square of beam current, we developed analysis methods that attempted to separate the two components, taking advantage of the fact that the phase stability of the coherent component allows for it to be subtracted from the total emission. (We initially adopted the term "phase stable" to describe this emission, since the degree of coherence was unknown.) To attempt to separate out the various components, we used two thicknesses ( $2 \mathrm{~mm}$ and $5 \mathrm{~mm}$ ) of tungsten radiators to convert the electron beam to bremsstrahlung photons, since this provided a different bremsstrahlung energy distribution and beam emissivity, which could modify the relative contributions of the two components. Our measurements were unable to separate out any significant incoherent signal component in these data, due primarily to background issues, and indicating that the coherent or phase-stable component was at least an order-of-magnitude larger.

In Fig. [5 (top), we show results of the AWA measurements at $20 \mathrm{GHz}$ (Ka-band) using the $5 \mathrm{~mm}$ radiator, which gave the cleanest background-subtracted results. Partially coherent emission was observed about $50 \mathrm{~ns}$ after beam passage. While the presence of the beam gave clear excess power levels, there is also considerable apparent "signal" present when the beam was blocked with lead just before our system. We found that acceleration and production of the electron beam within the of order $15 \mathrm{~m}$ length of this accelerator required that a major fraction ( $80 \%$ or more in some cases) of the electrons were removed by upstream collimation (a controlled scraping of the beam), but without any way to remove the secondary radiation (mostly hard bremsstrahlung photons) that this produced. This led to a high level of background ionization in both our chamber and the surrounding vault, leading to doubts about the reliability of the results. This is evident in Fig. 5, where the backgrounds with the beam blocked can be seen to at times exceed even the apparent signal. We confirmed the presence of such backgrounds using external ionization detectors. 
We also checked carefully whether any portion of these backgrounds could be due to radio-frequency interference, and we confirmed that this was not the case.

However, we note that our conclusion regarding these backgrounds implies that they are actually stray signal, due to the unwanted beam albedo (the beam components that caused scattered bremsstrahlung due to impacts with the side-walls of the beampipe). Thus it appeared to us that the presence of microwave emission from the chamber ionization was unavoidable. To further pursue the investigation with a more tightly controlled beam, another experiment was scheduled at The Stanford Linear Accelerator Center.

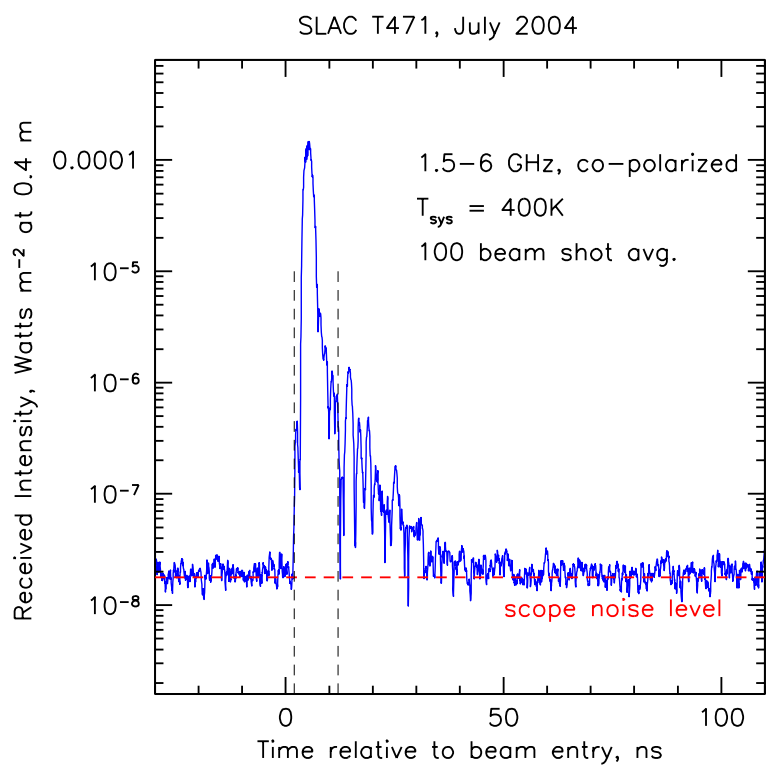

FIG. 6: Average microwave emission amplitude from 100 beam shots taken near shower-maximum in the 2004 SLAC T471 experiment, using a broadband antenna that was polarized along the electron beam axis, and was thus sensitive to partially coherent radiation directly from the relativistic electron shower as it transited the Faraday chamber. A strong initial pulse is seen, with rapid decay, followed by a second exponential tail with a longer decay. The noise level is in this case determined by the limited dynamic range of the oscilloscope used, rather than the thermal noise level.

\section{SLAC T471/E165.}

In the following year, a similar experiment, T471, was performed at the Stanford Linear Accelerator Center. The configuration of this experiment was largely the same as that of INCOBREMS, but additional precautions were taken against EMI and beam backgrounds, and verified in lab and beam calibration tests. This experiment was coordinated to be operated just downstream of the E165 FLASH experiment, which was used to do precise calibration of air fluorescence for the HiRes collaboration [32]. The SLAC T471/E165 experiments also used a precisely controlled, $28 \mathrm{GeV}$ electron beam which was collided with a target consisting of $90 \% \mathrm{Al}_{2} \mathrm{O}_{3}$ and $10 \% \mathrm{SiO}_{2}$

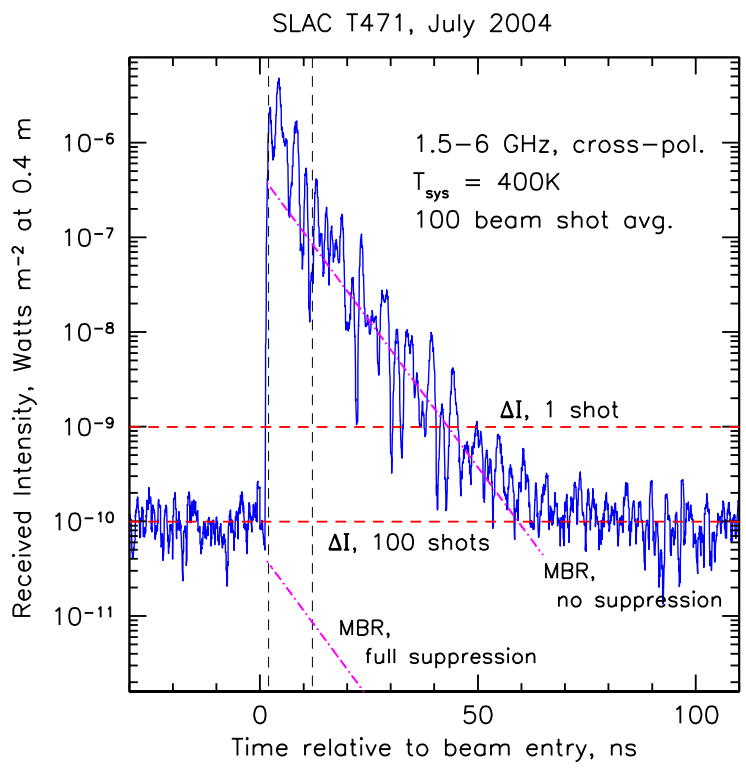

FIG. 7: A plot similar to the previous figure, but now using a crosspolarized antenna which was insensitive to radiation polarized with the electron beam. The dynamic range of the system was now improved so that the noise level is determined by thermal noise, and the detected microwave emission extends out to $60 \mathrm{~ns}$ or more, with an exponential decay time constant of about $7 \mathrm{~ns}$. The upper and lower dashed red horizontal lines indicate the minimum detectable intensity, as given by equation 8 for the single-shot case, and the 100shot average. The diagonal dot-dash lines are the two extreme-case estimates for MBR emission: the upper case for no net collisional suppression and the lower case for maximal collisional suppression of the emission, both for the case where the electron thermalization time constant is the source of the 7 ns exponential decay observed.

to make showers with varying particle number, from 0 to 14 radiation lengths of material. In T471/E165, the $28.5 \mathrm{GeV}$ electron bunches were used directly to create the showers with no intermediate conversion to photons via a bremsstrahlung radiator, as this was unnecessary given the high electron energy. Bunches with a typical charge of $\sim 2 \times 10^{7}$ electrons were used, giving a total shower energy of typically $6 \times 10^{17} \mathrm{eV}$, very similar to those used at AWA.

Figure 6 shows results from measurements of the emission over the $1.5-6 \mathrm{GHz}$ band, using an antenna that was co-polarized with the electron shower momentum. Here the square of the average signal voltage is plotted vs. the time after beam entry into the Faraday chamber. The transit time for the chamber is about $3.3 \mathrm{~ns}$ for the beam. An initial strong impulse is observed at the first causal point in time after beam entry. This impulse is found to be highly polarized with the plane of polarization aligned with the beam axis and Poynting vector, characteristic of transition and radio Cherenkov radiation. Such emission was anticipated, and is damped almost immediately due to the microwave absorber $(\geq 30 \mathrm{~dB}$ per reflection even at angles of order $55^{\circ}$ from normal incidence) that covers the interior of the Faraday chamber (the implied average time constant for quasi-exponential decay of reflec- 


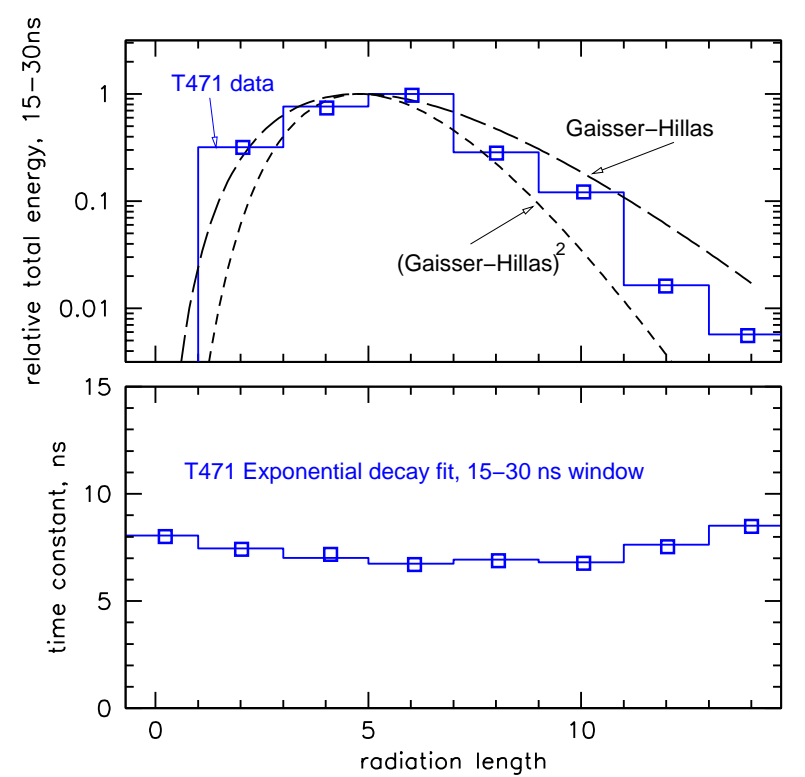

FIG. 8: Top: relative integrated energy in the tail of the microwave emission, between 15 to $30 \mathrm{~ns}$, as a function of the depth of the shower in radiation lengths. The curve shows a Gaisser-Hillas shower profile for comparison, which peaks at about 4.7 radiation lengths at shower maximum. By contrast, the microwave tail emission shows some early radiation probably due to the initial electron bunch. Near shower maximum, the shower charge overcomes the beam charge, and the emission appears to follow the shower profile near shower maximum, though it falls below the particle number profile at late times.

tions is of order $1.3 \mathrm{~ns}$ for this absorber in this geometry). The noise level in this plot is dominated by digitization noise, since the sensitivity had to be reduced in order to achieve enough dynamic range to see the strong initial impulse.

In Fig. (7) we plot the same measurements made with a crosspolarized antenna, which was therefore insensitive to the relativistic shower emission, with a $20 \mathrm{~dB}$ cross-polarization rejection factor. In this case the strong initial impulse is not prevalent though in fact the leading edge is likely to be slightly influenced by the $-20 \mathrm{~dB}$ leakage from the other polarization. The exponentially-decaying tail of emission extends out to $60 \mathrm{~ns}$ or more, with noise levels now determined by the thermal noise level rather than scope noise. Based on beam-out vs. beam-in and beam-on vs. beam-blocked measurements, no beam-related background (either ionization or EMI) was present, and thus no background subtraction was necessary. Several curves are also plotted with the data. The horizontal lines indicate the thermal noise level for single shots and for the average of the 100 beam shots used here, based on equation 8 above. The diagonal dot-dash curves are model predictions based on equation 7 above, calculated for the two extreme cases of the collisional term $\zeta_{c}$ from equation 3 , one for the case of no suppression $\left(\zeta_{c}=1\right)$ and the other for full collisional suppression $(\xi=1)$. It is evident that, if MBR is responsible for this emission, the collisional suppression is almost completely offset by the partial coherence.
In Fig. 8 top) we plot the behavior of the integrated microwave energy in the 15-30 ns window as a function of shower depth in radiation lengths. The emission from the direct beam, which adds noticeably to the shower emission up to about 4 radiation lengths, has been subtracted here in proportion to the depth in radiation lengths, so that the contribution from the shower emission alone can be compared to expectations. The upper (long-dashed) curve shown is a standard Gaisser-Hillas profile [40], peaking at about 4.7 radiation lengths for these showers. The lower (short-dash) curve is a Gaisser-Hillas profile, but now scaled as the square of the particle number in the shower. It is evident that the shower emission scales roughly with the particle number in the shower, but appears to drop below the standard Gaisser-Hillas profile at large shower depths, although not enough to warrant scaling that is quadratic in particle number. This behavior provides evidence that the process for the emission is relatively insensitive to the plasma density. At larger shower depths in particular the plasma density decreases by 1-2 orders of magnitude with only factors of 2-3 apparent drop in the relative microwave emission compared to expectation based on the standard Gaisser-Hillas profile.

Fig. 8 (bottom) shows that the fitted time constant of the decay of the emission power is roughly constant with shower depth at about $7 \mathrm{~ns}$, with some indication that it may be increasing for large shower depths. The near constancy of this parameter indicates that the underlying physical process that removes the radiating electrons from the emitting populations is nearly completely insensitive to plasma density.

The radiation observed in T471 is also partially coherent. This is shown in Fig. 9, which plots the integrated microwave power from 15-30 ns after the main pulse vs. beam energy as measured by an external transition radiation detector. The quadratic correlation here indicates that the partially coherent portion of the emission dominates completely over incoherent emission. The coherent emission appears to be several thousand times the expected incoherent emission level, implying that subgroups of $\mu_{e} \simeq 10^{3-3.5}$ electrons are radiating quasi-coherently within their subgroup, using the notation of equation 14 above. Given that the showers used in T471 created $3 \times 10^{13}$ ionization electrons within the Faraday chamber used, the net correlation of $\sim 10^{-10}$ is still extremely small, and it is evident that this level of partial coherence is very far removed from the full-spatial coherence that obtains in coherent Cherenkov or transition radiation.

We note that the Debye length (equation 11 above) for the T471 plasma is initially of order $2 \mathrm{~mm}$ when $T_{e} \simeq 10^{4.5} \mathrm{~K}$, and within one Debye radius there are of order $10^{7}$ free electrons initially. Thus a weak correlation of $\sim 0.01 \%$ within a Debye radius appears to be all that is required to create the observed partial coherence effects. This analysis does not account for the rapid evolution of the Debye length as the electrons cool however. At $T_{e}=10^{3} \mathrm{~K}$, close to ambient, $\lambda_{D} \simeq 0.3 \mathrm{~mm}$, and the Debye volume then contains of order $10^{5}$ electrons, still requiring only a $1 \%$ correlation coefficient. However, any prediction using a simple plasma-correlation model requires understanding of the dynamics of the cooling event before a self-consistent picture can emerge. 


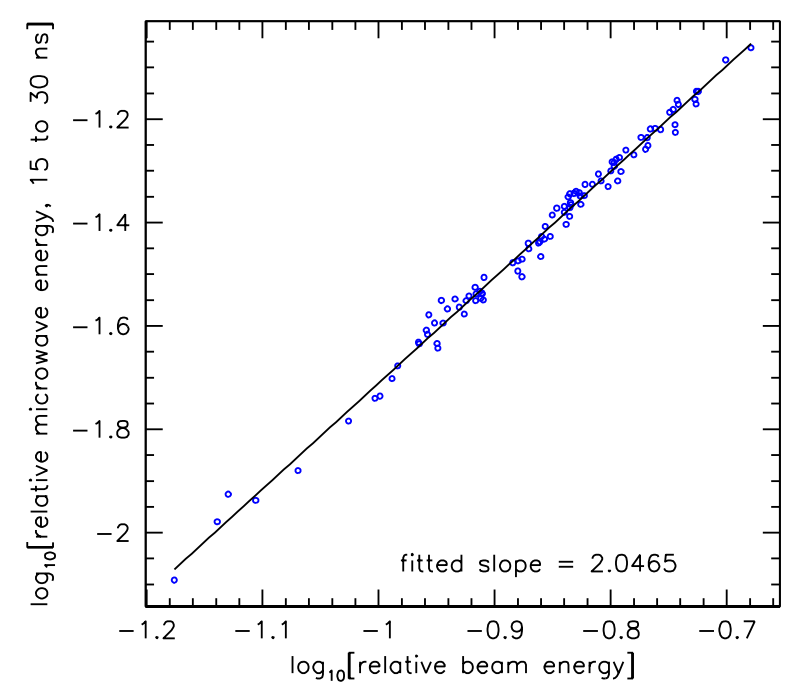

FIG. 9: Plot of relative microwave energy in the tail of the observed air plasma emission vs. the relative microwave energy observed in an external transition-radiation monitor of the beam current, which is directly proportional to beam energy. The observed microwave power follows closely a quadratic rise with beam energy, characteristic of coherent radiation.

\section{Scaling to air showers.}

Under the assumption that standard radiation scaling laws obtain, we can make an estimate of the threshold for detectability of the emission seen in Fig. 7, if we scale it to air shower observation distances and a realistic detection system.

To do this, we take the flux density as estimated from the data in Fig.7 using the weighted average T471 antenna effective area of $0.05 \mathrm{~m}^{2}$. Using this flux density and the equivalent shower energy of $3.4 \times 10^{17} \mathrm{eV}$, we scale to an equivalent air shower at a distance of $10 \mathrm{~km}$. The scaling corrects for the lower electron density expected for a typical $5 \mathrm{~km}$ air shower altitude. We also assume an integration time (several hundred ns) based on angular transit times for showers at roughly this distance, with the peak flux density determined by the emission over an interval comparable to the thermalization time. We consider both linear and quadratic scaling of the emission with electron density, and with regard to the shower geometry, as long as the transverse diameter of plasma column is contained within the antenna beam, we assume there is a direct scaling from the T471 observations to air shower observations.

The results of this analysis indicate that, if the partially coherent emission observed in T471 scales only linearly with shower energy (as might be expected in the pure "incoherent" case), then the threshold of the AMBER system at $10 \mathrm{~km}$ is of order $8 \times 10^{18} \mathrm{eV}$ in shower energy. If the scaling is quadratic with shower energy, as the data suggests, the threshold is lower, of order $1.6 \times 10^{18} \mathrm{eV}$.
We can also estimate the maximum distance to which a shower at the GZK threshold energy of $3 \times 10^{19} \mathrm{eV}$ could be observed under these same conditions: for the linear-scaling case, the distance is of order $20 \mathrm{~km}$; for the quadratic case it is much larger, of order $200 \mathrm{~km}$, but of course in this case earth-curvature and atmospheric attenuation would also require consideration. In either case, the current emission parameters strongly warrant further investigation of the potential for development of MBR detection of air showers.

As noted in a previous section, an air shower plasma can also be characterized in terms of its Debye length. For a $10^{19} \mathrm{eV}$ shower, with an initial electron density of order $10^{10}$ $\mathrm{e}^{-} \mathrm{m}^{-3}$ within a few $\mathrm{m}$ of its core, $\lambda_{D} \simeq 7 \mathrm{~cm}$ for $T_{e}=10^{4} \mathrm{~K}$ during the early period of the electron cooling, and $\lambda_{D} \simeq 1 \mathrm{~cm}$ once the electrons have cooled close to ambient levels. The electron number within a Debye radius similarly evolves from $\sim 10^{7}$ to $\sim 10^{4}$ over the same cooling period, several tens of ns. These values leave open the possibility of correlated electron behavior comparable to those seen in our T471 experiment, if the correlations are related to plasma density parameters.

\section{Beyond detection: Shower Calorimetry with an AMBER array.}

The importance of MBR detection of EAS rests in the potential that it will yield the observational advantages comparable to those of optical fluorescence without the shortcomings associated with weather and limited duty cycle. By observing MBR, one is observing an EAS from the same perspective as with optical fluorescence, via energy-loss processes that are intimately related to the excitation of molecular nitrogen that leads to air fluorescence. However, observations can occur 24 hours per day, and at the microwave bands of interest there is virtually no attenuation due to atmospheric contamination from aerosols or clouds. Even heavy rain leads to attenuation of $\leq 1 \mathrm{~dB}$ above elevation angles of $30^{\circ}$ at C-band $(4-6 \mathrm{GHz})$, a $20 \%$ effect. Initially, while the MBR technique is being crosscalibrated with respect to an optical fluorescence and ground array, this immunity to atmospheric effects can yield immediate benefits in helping to extrapolate the energy scale for distant events, where optical fluorescence is most affected by aerosols and other atmospheric uncertainties.

Commercially designed microwave reception equipment can be easily weatherproofed, and future arrays would most likely be able to employ off-the-shelf satellite television components, taking advantage of the tremendous economy of scale in wireless and satellite television technology. Following validation of the technique in coincidence with an existing EAS installation, MBR detectors could be potentially deployed as standalone UHECR observatories. Critical to the success of such an observatory is the ability of MBR to do precision shower calorimetry. There appears to be good initial evidence from the T471 experiment that such calorimetry can be done with precision that is comparable to current techniques.

Referring to Figure 9 we stress that individual singleshot measurements of the integrated microwave energy in the 
TABLE II: Detectability of air showers assuming that the T471 shower emission shown in Fig. 7 can be scaled to an air shower at $10 \mathrm{~km}$ distance, reaching maximum at $5 \mathrm{~km}$ altitude, and detected with a $1.8 \mathrm{~m}$ diameter dish at $4 \mathrm{GHz}$ (C-band) using standard satellite dish technology. Results are estimated both for linear and quadratic scaling with shower energy.

\begin{tabular}{|c|c|c|c|}
\hline Inputs and derived parameters & expression & value & units \\
\hline \multicolumn{4}{|l|}{ Observation parameters } \\
\hline peak intensity observed, T471, single pol & $\mathrm{I}_{0}$ & $1.00 \mathrm{E}-06$ & $\mathrm{~W} / \mathrm{m}^{\wedge} 2$ \\
\hline time constant & $\tau$ & $1.00 \mathrm{E}-08$ & $\mathrm{~s}$ \\
\hline reference bandwidth & $\Delta \mathrm{f}_{0}=\Delta \omega / 2 \pi$ & $2.50 \mathrm{E}+09$ & $\mathrm{~Hz}$ \\
\hline reference system temperature & $\mathrm{T}_{\text {sys, ref }}$ & $4.00 \mathrm{E}+02$ & $\mathrm{~K}$ \\
\hline reference flux density & $\mathrm{I}_{\mathrm{f} 0}=\mathrm{I}_{0} / \Delta \mathrm{f}_{0}$ & $4.00 \mathrm{E}-16$ & $\mathrm{~W} / \mathrm{m}^{2} / \mathrm{Hz}$ \\
\hline \multicolumn{4}{|l|}{ Reference shower parameters } \\
\hline electron energy & $\varepsilon$ & $2.80 \mathrm{E}+10$ & $\mathrm{eV}$ \\
\hline beam current & $\mathrm{j}_{\mathrm{b}}$ & $1.20 \mathrm{E}+07$ & e-/pulse \\
\hline observed reference shower length & $\mathrm{L}_{0}$ & 0.65 & $\mathrm{~m}$ \\
\hline distance to reference shower axis & $\mathrm{d}$ & 0.5 & $\mathrm{~m}$ \\
\hline total reference shower equivalent energy & $\mathrm{E}_{0}=\varepsilon \mathrm{j}_{\mathrm{b}}$ & $3.36 \mathrm{E}+17$ & $\mathrm{eV}$ \\
\hline \multicolumn{4}{|l|}{ Parameters for estimation of trial air shower signal } \\
\hline Trial distance to air shower & $\mathrm{R}$ & $1.00 \mathrm{E}+04$ & $\mathrm{~m}$ \\
\hline mean altitude of trial shower maximum & $\mathrm{a}$ & $5.00 \mathrm{E}+00$ & $\mathrm{~km}$ \\
\hline Trial dish diameter & $\mathrm{D}$ & 1.8 & $\mathrm{~m}$ \\
\hline feed efficiency & $\eta$ & 0.8 & \\
\hline dish effective area & $A_{\text {eff }}=\eta \pi(D / 2)^{2}$ & 2.036 & $\mathrm{~m}^{2}$ \\
\hline center frequency of observation & $f_{c}$ & $3.80 \mathrm{E}+09$ & $\mathrm{~Hz}$ \\
\hline receiver bandwidth & $\Delta \mathrm{f}$ & $5.00 \mathrm{E}+08$ & $\mathrm{~Hz}$ \\
\hline shower length over 1 e-folding decay time & $\mathrm{L}_{\tau}=\mathrm{c} \tau$ & $3.00 \mathrm{E}+00$ & $\mathrm{~m}$ \\
\hline $\begin{array}{l}\text { Ratio of electron density at shower altitude to sea level } \\
\text { electron density in reference shower }\end{array}$ & $\rho$ & $6.00 \mathrm{E}-01$ & \\
\hline $\begin{array}{l}\text { Ratio of trial shower length per e-folding time to } \\
\text { observed reference shower length }\end{array}$ & $\Gamma=\mathrm{L}_{\tau} / \mathrm{L}_{0}$ & 4.62 & \\
\hline Expected signal flux density & $\mathrm{I}_{\mathrm{f}, \exp }=\mathrm{I}_{\mathrm{f} 0} \Gamma \rho(\mathrm{R} / \mathrm{d})^{-2}$ & 2.77E-24 & $\mathrm{W} / \mathrm{m}^{2} / \mathrm{Hz}$ \\
\hline \multicolumn{4}{|l|}{ Parameters for estimate of minimum detectable flux density } \\
\hline feed+dish beamwidth on sky & $\Delta \theta=1.22 \mathrm{c} /(\mathrm{f} \mathrm{D})$ & 5.35E-02 & $\mathrm{rad}$ \\
\hline observed average shower length & $\mathrm{L}_{\mathrm{av}}=\mathrm{R} \Delta \theta$ & $9.90 \mathrm{E}+02$ & $\mathrm{~m}$ \\
\hline $\begin{array}{l}\text { average duration of emission in field of view = } \\
\text { integration time }\end{array}$ & $\Delta \mathrm{t}=\mathrm{L}_{\mathrm{av}} / \mathrm{c}$ & $3.27 E-06$ & $\mathrm{sec}$ \\
\hline System temperature & $\mathrm{T}_{\mathrm{sys}}$ & $8.00 \mathrm{E}+01$ & $\mathrm{~K}$ \\
\hline Boltzmann's constant & $\mathrm{k}$ & $1.38 \mathrm{E}-23$ & $\mathrm{~W} / \mathrm{K}$ \\
\hline minimum detectable flux density & $\Delta \mathrm{I}=\mathrm{k} \mathrm{T} \mathrm{sys} /\left[\mathrm{A}_{\mathrm{eff}}(\Delta \mathrm{t} \Delta \mathrm{f})^{1 / 2}\right]$ & $1.34 \mathrm{E}-23$ & $\mathrm{~W} / \mathrm{m}^{2} / \mathrm{Hz}$ \\
\hline \multicolumn{4}{|l|}{ SNR and predicted threshold energy } \\
\hline signal to noise ratio for reference shower at $R$ & $\mathrm{SNR}_{0}=\mathrm{I}_{\mathrm{f}, \exp } / \Delta \mathrm{I}$ & $2.06 \mathrm{E}-01$ & \\
\hline $\begin{array}{l}\text { Threshold shower energy required for } 5 \sigma \text {, quadratic } \\
\text { power dependence with shower energy }\end{array}$ & $\mathrm{E}_{\text {thresh }}=\left(5 / \mathrm{SNR}_{0}\right)^{1 / 2} \mathrm{E}_{0}$ & $1.65 \mathrm{E}+18$ & $\mathrm{eV}$ \\
\hline $\begin{array}{l}\text { Threshold shower energy required for } 5 \sigma \text {, linear power } \\
\text { dependence with shower energy }\end{array}$ & $\mathrm{E}_{\text {thresh }}=\left(5 / \mathrm{SNR}_{0}\right) \mathrm{E}_{0}$ & $8.14 \mathrm{E}+18$ & $\mathrm{eV}$ \\
\hline $\begin{array}{l}\text { Maximum distance a } 3 \times 10^{19} \mathrm{eV} \text { shower could be } \\
\text { observed, assuming linear scaling }\end{array}$ & $\mathrm{R}_{\max }=\left(3 \times 10^{19} / \mathrm{E}_{\text {thresh }}\right)^{1 / 2} \mathrm{R}$ & $1.92 \mathrm{E}+04$ & $\mathrm{~m}$ \\
\hline $\begin{array}{l}\text { Maximum distance a } 3 \times 10^{19} \mathrm{eV} \text { shower could be } \\
\text { observed, assuming quadratic scaling }\end{array}$ & $\mathrm{R}_{\max }=\left(3 \times 10^{19} / \mathrm{E}_{\text {thresh }}\right) \mathrm{R}$ & $1.81 \mathrm{E}+05$ & $\mathrm{~m}$ \\
\hline
\end{tabular}




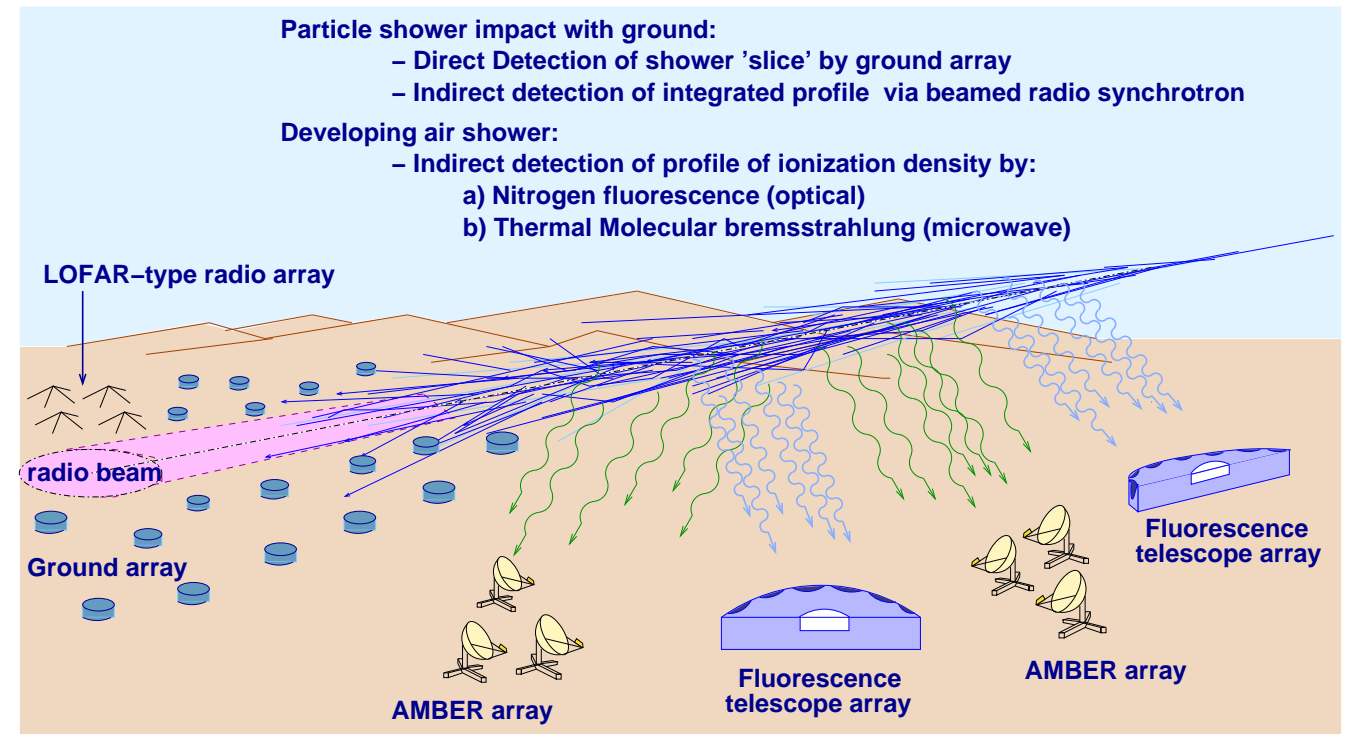

FIG. 10: Conceptual sketch of how microwave bremsstrahlung detection relates to other methods of ultra-high energy cosmic ray air shower detection.

shower can be used to determine the beam energy in the experiment to about $2-3 \%$ precision, once the overall energy scale is set (in this case by an external beam current monitor). This level of precision is set entirely by the instantaneous signal-tonoise of the data, and is not systematics-limited, as evidenced by the precise quadratic scaling observed. In the T471 experiment, this scaling was observed over more than a decade (not shown in Fig. 9) of energy. In addition, the same scaling was seen at many different relative plasma densities (created by sampling the shower at different depths of development) in the experiment, indicating that it is not dependent on shower age. Such results give us good confidence that, with sufficient attention to careful detector and system design, and adequate calibration, an MBR observatory could provide shower calorimetry which was comparable to that of existing techniques.

EAS observatories have also demonstrated capabilities for neutrino observations, but will require substantial increases in their apertures before such techniques can become practical in elucidating the GZK neutrino spectrum. Neutrino-induced showers are also likely to be highly-inclined relative to typical proton showers, and thus become problematic for observation with ground arrays, which suffer from more severe systematics at high zenith angles. In contrast fluorescence methods (and possibly the MBR methods we describe here) can readily observe such showers, since the geometry is no less favorable for horizontal than for vertical showers. Thus MBR observations may help to greatly expand the neutrino apertures of air shower observatories, by extending the duty cycle for "quasi-fluorescence" observations, perhaps by an order of magnitude or more. Figure 10 gives a schematic view of how such methodology relates to other implementations of ultrahigh enery cosmic ray air shower detection.

\section{The AMBER system.}

Following the indications of stronger-than-expected emission from the two accelerator experiments detailed above, we have moved ahead to develop a prototype of a system that could be used to search for detectable microwave emission from actual air showers. This system is built around a custom compact-PCI digitizer and data acquisition system, which we designate the Radio Bremsstrahlung Impulse Detector (RaBID). We have chosen the components and size of the prototype system such that it can be duplicated at low cost with mostly commercial parts. The proposed system, incorporating the RaBID prototype, is designated the AMBER for Airshower Microwave Bremsstrahlung Experimental Radiometer. AMBER is currently operating on the rooftop of Watanabe Hall at the University of Hawaii at Mānoa (UHM) in Honolulu, Hawaii, pictured in Fig. 11.

In its current configuration, the AMBER unit consists of a dual-band ( $\mathrm{C}$ and $\mathrm{Ku}$ ), dual-polarization feed horn array at the prime focus of a $1.8 \mathrm{~m}$ off-axis parabolic dish. The array is in a diamond-shaped configuration where each feed is $\sim 5.2^{\circ}$ from its nearest neighbor. Each feed produces four channels of signal which are amplified and down-converted in Low Noise Blocks (LNB) and then conveyed to the RaBID DAQ via RG11 coaxial cable, as shown in Figure 11. The RaBID DAQ consists of a pair of RaBID cards located inside a compact PCI (cPCI) crate, along with a cPCI CPU for data collection and logging. At the RaBID card input, the downconverted LNB outputs are measured with RF power monitor (MAX4003) chips, which provide output proportional to the received RF power, with approximately $70 \mathrm{~ns}$ integration time. This power level is sampled with a $32 \mathrm{MSa} / \mathrm{s}$ ADC and processed inside a Field Programmable Gate Array.

These digitized samples are processed in 3 parallel paths: (1) all C-band samples are logged into a hardware histogram- 


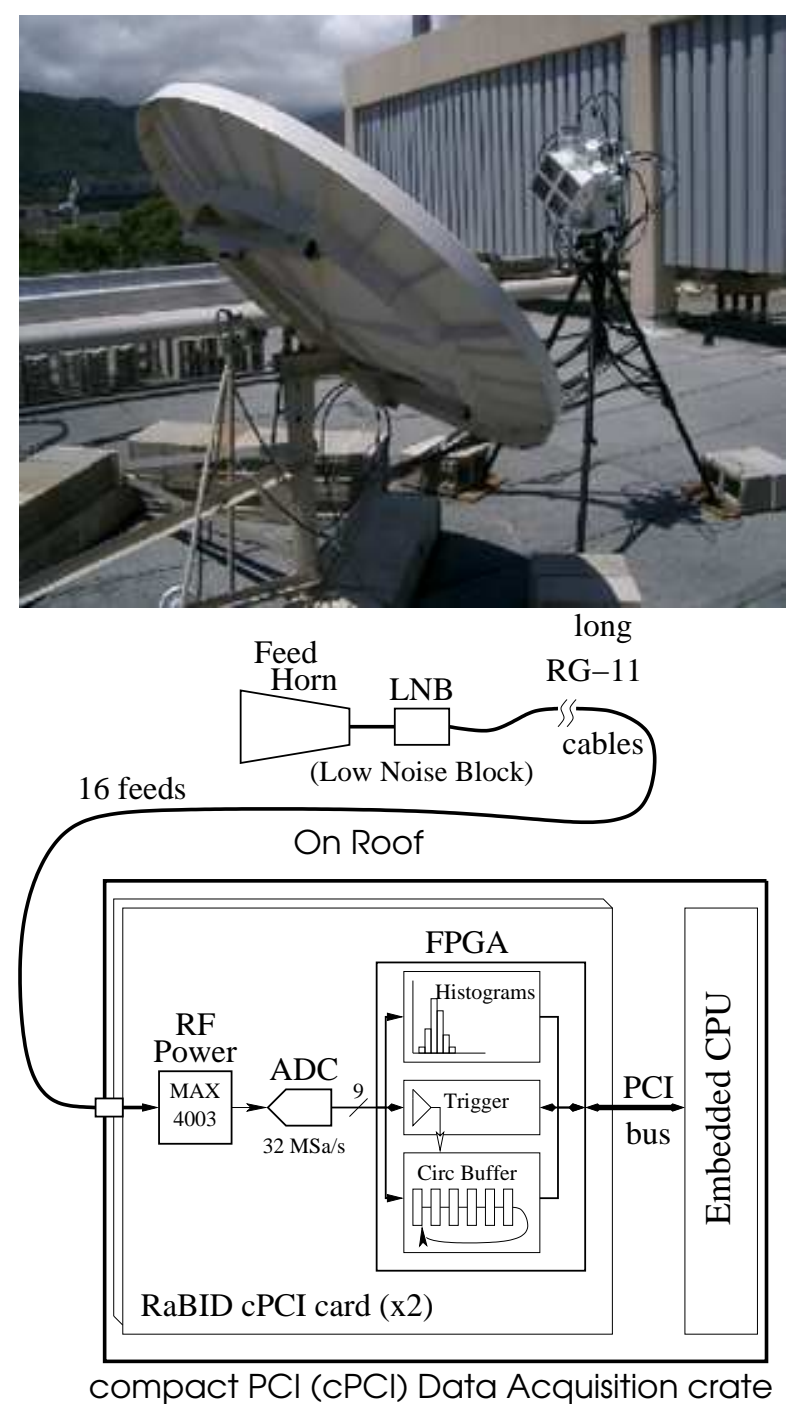

FIG. 11: Top: Prototype AMBER telescope and feed array on the roof of the physics building at UHM. Bottom: AMBER detector readout chain. The feed horn signals are amplified and down-converted in a Low Noise Block and then transmitted to a pair of RaBID cards for processing. See text for details.

mer, which allows optimum threshold-riding with varying background (2) a trigger threshold is set based upon the histogram values; and (3) a circular buffer holds the samples in time sequence to be read out into the CPU upon detection of a trigger condition. In order to avoid biases in the triggering, each feed horn channel (of 12 total) is triggered separately, at minimum possible threshold, and the trigger times (corresponding to different transit times across the array field of view) are analyzed in the stored data. All sample times are recorded with respect to a common clock, which is synchronized to GPS via Network Time Protocol. An external trigger port (not shown) is available for forcing readout when observing in conjunction with another detector.

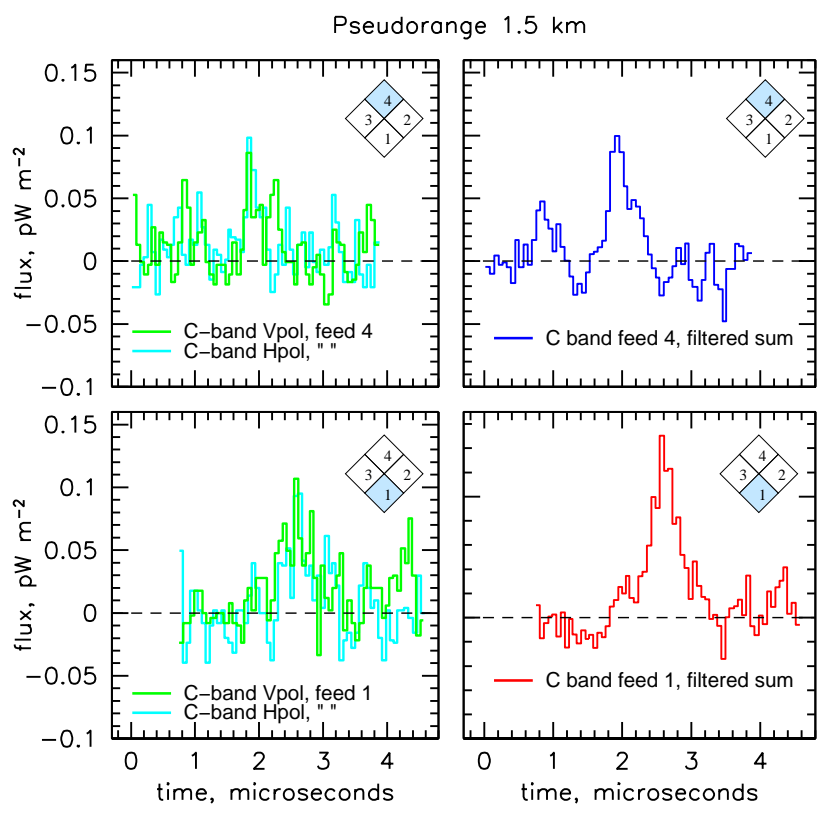

FIG. 12: Example of an event recorded recently with the prototype RaBID system in Hawaii. The two events occurred sequentially in the two feeds noted, and triggered both polarizations at both feed 4 (top) and feed 1 (bottom), creating a downgoing event trigger. Left,: Raw data, showing peaks in both $H$ and $V$ polarizations for the $C$-band feeds. Right: Signal after co-adding the polarizations and applying Wiener filtering to remove the high frequency thermal noise fluctuations. The pseudorange is based on the $680 \mathrm{~ns}$ dual-feed crossing time.

\section{AMBER Results.}

Since initial commissioning of the AMBER system began in mid-2005, we have accumulated about 8 months of data under stable operating conditions, most of which has been analyzed to search for EAS-like events. Because AMBER lacks a ground-truth EAS array to validate any observed signals, any candidates that are observed remain only putative at best. However, we may test a sample of such candidates for similarity to expectations from our simulations, and we have done this for a large data sample taken through the spring of this year, with results that support the potential for EAS measurements by an AMBER array.

Data analysis for AMBER events involves several steps which significantly improve the signal-to-noise ratio of the raw data. First, because the MBR signal is by nature unpolarized, we can combine the recorded power in the two independent polarizations, improving the SNR by a factor of order $\sqrt{2}$. Also, the signal arrives over many sequential $70 \mathrm{~ns}$ time bins, whereas a significant fraction of the noise is broadband and largely uncorrelated between successive time bins. These statements are equivalent to saying that the spectral bandwidth of the signal is much less than that of the noise, and under such conditions we may apply Wiener filtering (also known as $o p$ timal filtering) to remove the out-of-band noise component, and properly weight the in-band noise components. 

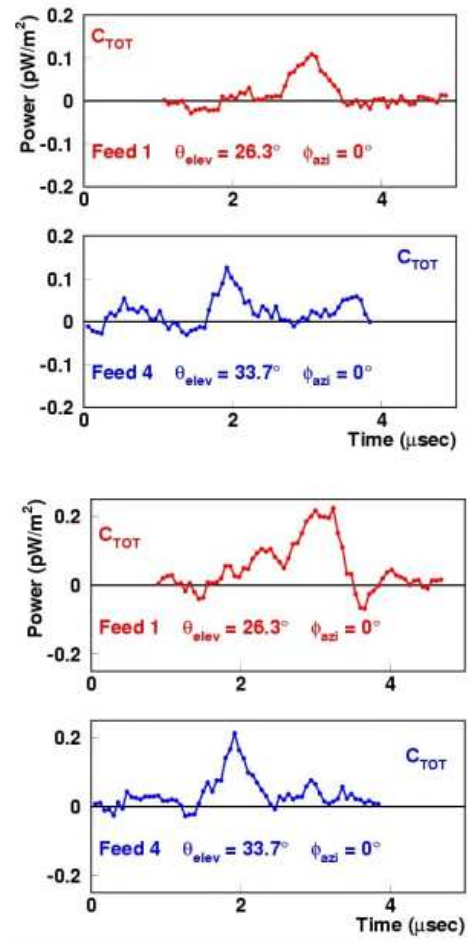
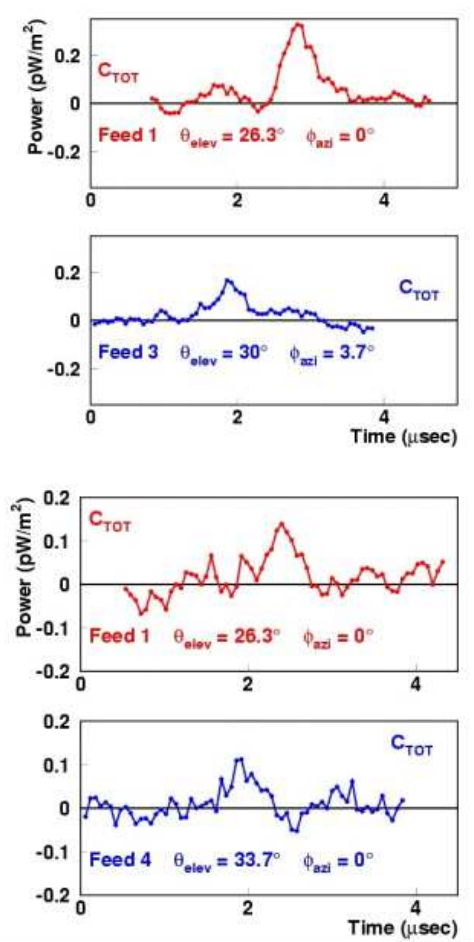

FIG. 13: Examples of other events recorded by the prototype AMBER system, meeting the criteria for EAS candidates. (In these data, the convention is reversed compared to Fig. 12, with the lower curve for each event originating from a feed which scans a higher elevation in the sky.)

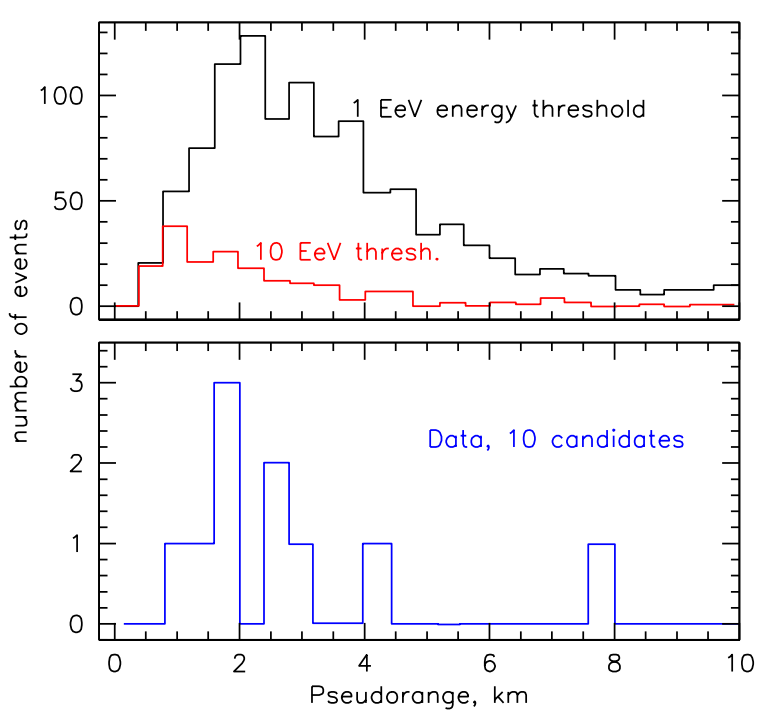

FIG. 14: Upper: Pseudorange distributions of simulated events for an AMBER array with a $1 \mathrm{EeV}$ energy threshold at $10 \mathrm{~km}$ distance (upper curve) and a $10 \mathrm{EeV}$ threshold at $10 \mathrm{~km}$ (lower curve). Lower: Pseudorange distribution of 10 candidate events measured in recent AMBER data taken over several months.

Examples of the effects of this analysis are shown for a candidate event in Fig. 12 On the left side the raw event that triggered the system at $\mathrm{C}$-band $(4 \mathrm{GHz})$ is shown, with the upper (earlier) signals from feed 4 and the later signals from feed 1 (lower left), indicating a downgoing event. On the right-side panes, the signals are shown for the combination of polarization co-adding and Wiener-filtering, with a marked improvement in overall SNR and resulting timing. Although the actual range to the event cannot be determined directly, we calculate a pseudorange based on the assumption that the feed-crossing signals were moving at the speed of light over the known angle between feeds. These pseudorange values can then be compared to simulations for actual EAS events.

Over the several months' observation period where the data has the highest quality, we have selected a sample of candidate events based on criteria derived from EAS expectations. Additional examples of such candidates are shown in Fig.13. Here the projected elevations and azimuths for each feed are shown in each event pair. We find that downgoing events predominate in our current sample. This is expected from a true EAS sample, but without an independent air-shower tag for any given event, we cannot yet reject the possibility of anthropogenic origin.

We can however compare the derived pseudorange distributions for both simulations and actual data to determine if the candidates observed in our event sample are drawn from a distribution that is consistent with what is expected from actual EAS events. To do this we have developed a Monte Carlo simulation code from which we can extract the pseudorange value for events with various detectability thresholds and an 
energy spectrum consistent with the known UHECR energy spectrum.

Results from this analysis are shown in Fig. 14. The upper pane shows the simulations for two energy threshold at a distance of $10 \mathrm{~km}$, and the lower pane shows the results for a sample of current candidate events. While these candidates cannot be proven to be EAS events without independent evidence from an air shower array, they do appear at least consistent to first order with the expectations from EAS events, and they demonstrate that an AMBER array has the basic detector characteristics to make measurements that are necessary to establish MBR observations as a viable EAS detection methodology.
In summary, we have proceeded as far as possible with MBR studies in the absence of coincident EAS ground-truth array measurements. Efforts are now underway to deploy an AMBER testbed array within the Auger Observatory in Argentina.

We thank the excellent support from staff at both Argonne National Laboratory, and the Stanford Linear Accelerator Center, especially the Experimental Facilities Division at SLAC. This work was supported through the Department of Energy High Energy Physics Division at the major laboratories, through DOE Outstanding Junior Investigator Awards to P. Gorham and D. Saltzberg, and through the DOE High Energy Physics Division University Program.
[1] J.Linsley, "Evidence for a primary cosmic ray particle with energy $10^{20}$ eV," Phys. Rev. Lett. 10 (1962) 146. First event observed and qualified as having reached the symbolic limit of $100 \mathrm{EeV}$.

[2] J. W. Cronin, Nucl. Phys. Proc. Suppl. 138, 465 (2005) [arXiv:astro-ph/0402487].

[3] D. J. H. Chung, G. R. Farrar and E. W. Kolb, Phys. Rev. D 57, 4606 (1998) |arXiv:astro-ph/9707036].

[4] T. Stanev, Astrophys. J. 479, 290 [arXiv:astro-ph/9607086].

[5] F. W. Stecker and S. L. Glashow, Astropart. Phys. 16, 97 (2001) [arXiv:astro-ph/0102226].

[6] M. Takeda et al., Phys. Rev. Lett. 81, 1163 (1998) [arXiv:astro-ph/9807193].

[7] R. U. Abbasi et al. [High Resolution Fly's Eye Collaboration], Phys. Rev. Lett. 92, 151101 (2004) [arXiv:astro-ph/0208243].

[8] K. Greisen, Phys. Rev. Lett. 16, 748 (1966).

[9] G. T. Zatsepin and V. A. Kuzmin, JETP Lett. 4, 78 (1966) [Pisma Zh. Eksp. Teor. Fiz. 4, 114 (1966)].

[10] R. U. Abbasi et al. [The High Resolution Fly's Eye Collaboration], Astrophys. J. 622, 910 (2005) [arXiv:astro-ph/0407622].

[11] T. Abu-Zayyad et al. [HiRes-MIA Collaboration], Astrophys. J. 557, 686 (2001) [arXiv:astro-ph/0010652].

[12] R. U. Abbasi et al. [The High Resolution Fly's Eye Collaboration], Astrophys. J. 623, 164 (2005) arXiv:astro-ph/0412617].

[13] A.V.Glushkov et al., Astropart. Phys. 4 (1995) 15.

[14] M.A. Lawrence, R.J.O. Reid, A.A. Watson. J. Phys. G. 17 (1991) 733.

[15] S.Yoshida et al., Astropart. Phys. 3 (1995) 105; also Shigeru Yoshida, Hongyue Dai, (astro-ph/9802294). Journal of Physics G 24 (1998) 905.

[16] D.J.Bird et al., Astrophys. J. 441 (1995) 144. J.W.Elbert, P.Sommers, Astrophys. J. 441 (1995) 151; Baltrusaitas, R.M., Cassiday, G.L., Elbert, J.W., et al., Phys. Rev. D 31, 2192 (1985).

[17] R. Abbasi et al. [HiRes Collaboration], Astropart. Phys. 21, 111 (2004) |arXiv:astro-ph/0309457].

[18] R. U. Abbasi et al., Astropart. Phys. 23, 157 (2005).

[19] R. Engel, D. Seckel, and T. Stanev, Phys. Rev. D 64, 093010 (2001).
[20] R. J. Protheroe \& P. A. Johnson, Astropart. Phys. 4, 253 (1996).

[21] O. E. Kalashev, V. A. Kuzmin, D. V. Semikoz and G. Sigl, Phys. Rev. D 66, 063004 (2002).

[22] R. U. Abbasi et al. [The High Resolution Fly's Eye Collaboration (HIRES)], Astrophys. J. 610, L73 (2004) |arXiv:astro-ph/0404137|.

[23] Y. Uchihori, M. Nagano, M. Takeda, M. Teshima, J. LloydEvans and A. A. Watson, Astropart. Phys. 13, 151 (2000) |arXiv:astro-ph/9908193|.

[24] S. C. Corbato et al., Nucl. Phys. Proc. Suppl. 28B, 36 (1992).

[25] J. W. Cronin et al., FERMILAB-PROPOSAL-0881

[26] P. Sommers [Pierre Auger Collaboration], arXiv:astro-ph/0507150

[27] R. O. Hundley, Tech. Rep. RM-3334-ARPA, The RAND Corporation (1962).

[28] G. Bekefi, Radiation Processes in Plasmas (Wiley, New York, 1966).

[29] A. Rosenberg, J. Felsteiner, Y. Ben-aryeh, \& J. Politch, Phys. Rev. Lett. 45, 1787, (1980).

[30] 2 A. Rosenberg, Y. Ben-aryeh, J. Felsteiner, \& J. Politch, Phys. Rev. Lett. 49, 1917, (1982).

[31] A. Rosenberg, J. Felsteiner, Y. Ben-aryeh, \& J. Politch, J. Appl. Phys. 60, (1986), 559.

[32] J. Belz et al. 2005, http://arxiv.org/abs/astro-ph/0507379

[33] R. H. Dicke, "The measurement of thermal radiation at microwave frequencies," Rev. Sci. Instrum. 17, (1946).

[34] H. Singh \& D. B. Graves, J. Appl. Phys. 87. (2000), 4098.

[35] U. Kortshagen, I. Pukropski, \& M. Zethoff, J. Appl. Phys. 70, (1994), 2040.

[36] B. L. Tembe and A. Mozumber, J. Chem. Phys. 78, 2030 (1983).

[37] J. M. Warman, M. Zhou-lei, and D. van Lith, J. Chem. Phys. 81, 3908 (1984).

[38] W. Sun, et al. Phys. Rev. A 52, 1229, (1995).

[39] R. D. Hake, Jr. and A. V. Phelps, Phys. Rev. 158, 70, (1967).

[40] T.K. Gaisser and A.M. Hillas, Proceedings of the 15th International Cosmic Ray Conference, 8, 353 (1977).

[41] Y. P. Raizer, Gas Discharge Physics, (Springer: Berlin), 1997.

[42] J. W. Goodman, Statistical Optics, (Wiley: New York), 1985. 